investigation of the chromatic effects in their receiving system was incomplete.

The possibility of making accurate spectral index measurements over small percentage bandwidths $(0.3$ per cent in this case) has several interesting applications. The non-thermal radiation from some radio sources may show changes in spectral index over small ranges of frequency as a result of complex spatial structure, self absorption, complexities in the energy spectrum of the relativistic electrons or the action of different radiation processes. Measurements of changes in the spectral index of variable radio sources at centimetre wavelengths would also be interesting. Finally, searches for radio spectral lines having large widths and poorly known frequencies could be facilitated with the measurement methods used here.

A more complete description of the measurements is in preparation, along with the results of further obscrvations.

I. thank Dr E. J. Blum and the radio astronomy groups at the Observatory of Paris and at Nancay for their help, and especially Mr R. Lauqué for assistance with receiver modifications. I acknowledge the financial support of a postdoctoral fellowship from the National Research Council of Canada.

Observatoire de Paris,

$$
\text { R. J. Alimen }
$$

Meudon, France.

Received August 5, 1968.

' Koehler, J. A., and Robinson, B. J., Astrophys. J., 146, 488 (1966).

${ }^{2}$ Blum, E. J., Boischot, A., and Lequeux, J., Proc. IRE Australia, 24, 208 (1963).

${ }^{3}$ Blum, F. T., Delannoy, J., LeRoux, E., and Weliachew, L., CR Acad. Sci.
Paris, 262, 1640 (1966).

Field, G. B., Astrophys. J., 135, 684 (1962).

${ }^{6}$ Lequeux, J., Ann. d'A strophys., 25, 221 (1962).

'Macdonald, G. H., Kenderdine, S., and Neville, A. C., Mon. Not. Roy. Astro. Soc., 138, 259 (1968).

? Ruze, J., Proc. IEEE, 54, 633 (1966).

\section{Pulsars and a Possible New Test of General Relativity}

HoFFMANN ${ }^{1-3}$ has suggested comparison of the frequency of terrestrial clocks and of signals from pulsars to test the prediction from general rolativity theory regarding the effect on frequency of changing gravitational fields and motion. Sadeh ${ }^{4}$ is reported to be making such a comparison. I wish to point out an amendment to Hoffmann's analysis.

For clocks on the Earth, Hoffrnann considers the possible effects of: (i) the variable gravity field of the Sun which is a maximum at perihelion and a minimum at aphelion; (ii) the gravity field of the 'Earth assumed constant at a given clock; (iii) the motion of the Earth around the Sun at variablo orbital velocity; and (iv) the rotation of the Farth about its axis with constant angular velocity.

In the approximation used, various effects are linearly superposed, and effect (iv), being constant, is neglected. Effect (ii) on a terrestrial clock is only an order of mag. nitude lower than (i), but because it is constant it may also be neglected if the effects of gravitational fields can be superposed. Hoffmann ${ }^{3}$ has shown that the velocity effect (iii) augmonts the gravity effect (i). Ho considers the Schwarzschild line-element-the proper-time differential $\mathrm{d} s$ recorded by a clock carried on the Eerth, which is given by the equation

$$
\begin{gathered}
\mathrm{d}^{2} s=\left(1-2 M G / c^{2} r\right) \mathrm{d} t^{2}-\left(1-2 M G / c^{2} r\right)^{-1} \mathrm{~d} r^{2} / c^{2}- \\
r^{2}\left(\mathrm{~d} \theta^{2}+\sin ^{2} \theta \mathrm{d} \varphi^{2}\right) / c^{2}
\end{gathered}
$$

Here $M$ is the mass of the Sun, $a$ the gravitational constant, $c$ the velocity of light, $r$ the curvature coordinate for the path of the Earth with the centre of the Sun as the origin of spatial coordinates $5, t$ the proper time for a clock fixed at $r=\infty^{5}$, and $\theta$ and $\varphi$ are the polar angles corresponding to $r$. Cgs units are used.

Using suffixes $p$ and $a$ for perihelion and aphelion, and noting that $\mathrm{d} r / \mathrm{d} t=0$ at perihelion and aphelion; $\mathrm{d} \varphi / \mathrm{d} t=$ $v / r$, where $v$ is the orbital velocity; $\theta=\pi / 2$ for orbit in a plane; and $v^{2}=M G / r$, Hoffmann obtains the following result for the Earth clock at perihelion and aphelion

or

$$
\mathrm{d} s_{p} / \mathrm{d} s_{a} \sim\left(1-3 M G / 2 c^{2} r_{p}\right) /\left(1-3 M G / 2 c^{2} r_{a}\right)
$$

$$
\left(\mathrm{d} s_{p} / \mathrm{d} s_{a}-1\right) \sim-3 M G\left(r_{a}-r_{p}\right) / 2 c^{2} r_{a} r_{p}
$$

The two rates aro found to differ by $4.9 \times 10^{-10}$ in 1 , assuming that the astronomical observations for the distances at perihelion and aphelion correspond to $r_{p}$ and $r_{a}$.

The relation $v^{2}=M G / r$ holds only for a circular orbit, and if the equation for an elliptical orbit $\left(v^{2}=2 M G / r-\right.$ $2 M G /\left(r_{a}+r_{p}\right)=2 M G / r$ - a constant, neglecting the mass of the Earth) is used instead, the numerical result is $6.6 \times 10^{-10}$ instead of $4.9 \times 10^{-10}$. It is, however, desirable and possible to work wholly within the general theory of relativity, instead of using an amalgam of Newtonian and Einstein's gravitational theories. The Earth follows a geodetic path in the Schwarzschild field of the Sun, and the following result is obtained from the usual (general) relativistic theory of planetary orbits ${ }^{6}$.

$$
\mathrm{d} s / \mathrm{d} t=\text { constant } \times\left(1-2 M G / c^{2} r\right)
$$

and $\mathrm{d} s_{p} / \mathrm{d} s_{a}=\left(1-2 M G / c^{2} r_{p}\right) /\left(1-2 M G / c^{2} r_{a}\right)$, now exactly. Then

$$
\left(\mathrm{d} s_{p} / \mathrm{d} s_{a}-1\right) \sim-2 M G\left(r_{a}-r_{p}\right) / c^{2} r_{a} r_{p}
$$

This is $4 / 3$ of the result obtained by Hoffmann, the difference in time rate being $6.6 \times 10^{-10}$.

A similar analysis would apply to intrinsic variations in the frequency of signals from pulsars, should they be orbiting systems.

In this derivation, I have followed the classical theory used in determining the advance of perihelion of Mercury. A more exact treatment dealing with the Earth, the Moon and the Sun as a three body system and taking into account variations in the mass of the Earth and the Moon in their orbits is not likely to yield a substantially different result.

It scems that the Earth in pursuing a geodetic path around the Sun is in a state of "free fall", and a naive application of the principle of equivalence, in this sense, would suggest that there is no resultant effect of the changes in the gravity field of the Sun, and in the consequent acceleration and motion of the Earth, on the rate of terrestrial clocks. But the validity of the principle of equivalence in this sense is doubtfiul?

11-A Koregaon Park,

(*. H. Keswant

Poona 1, India.

Received .July 23; revised Angust 20, 1968.

${ }^{1}$ Hofimann, B., Nature, 218, 667 (1968).

${ }^{2}$ Hoffmann, B., Nature, 218, 757 (1968).

${ }^{3}$ Hofrmunn, B., and Sproull, W. T., Amer. J. Phys., 29, 640 (1961).

${ }^{4}$ Time, 29 (June 14, 1968). synge, J. L., Relativity: The General Theory, 268 (North-Holland Pub.
Co, Amsterdam, 1960).

- Eddington, A. S., The Mathematical Theo'y of Relativity, 86 (Cambridge, 1930 ).

${ }^{2}$ Keswani, G. H., Brit. J. Phil. Sci., 16, 273 (1966).

\section{Superconductivity in White Dwarfs and Pulsars}

IT is quite possible, though not proved, that pulsars are magnetic white dwarf stars ${ }^{1}$. On the other hand, none of the four known pulsars has been identified with a white dwarf-the star which seemed to have been identified with $C P 1919$ is not optically variable and, besides, is 